\title{
Feed Forward Networks in Color Extended Visual Cryptography to Generate Meaningful Shares
}

\author{
J. Ida Christy and V. Seenivasagam \\ ${ }^{1}$ Associate Professor in CSE Dept., \\ Unnamalai Institute of Technology \\ Kovilpatti, India \\ ${ }^{2}$ Professor in CSE Dept., \\ National Engineering College, \\ Kovilpatti, India \\ idaselvin@gmail.com,yespee1094@yahoo.com
}

\begin{abstract}
Conventional visual secret sharing schemes generate noise-like random pixels on shares to hide secret images. It suffers a management problem, because of which dealers cannot visually identify each share. This problem is solved by the Extended Visual Cryptography Scheme (EVCS), which adds a meaningful cover image in each share. However, the previous approaches involving the EVCS for color images suffer from a pixel expansion problem. In this paper, we propose a general approach to solve the abovementioned problem; the approach can be used for color images in non computer-aided decryption environments. The proposed approach uses Feed Forward Network to construct the shares. The experimental results indicate that a solution to the pixel expansion problem of the EVCS is achieved. Moreover, the display quality of the recovered image is better than that obtained using other $V C$ schemes.
\end{abstract}

Keywords: Visual Secret Sharing, Extended Visual Cryptography (EVC), Feed Forward Network, Shares, Pixel Expansion

\section{Introduction}

Today most applications are only as secure as their underlying system. Since the design and technology of middleware has improved steadily, their detection is a difficult problem. As a result, it is nearly impossible to be sure whether a computer that is connected to the internet can be considered trustworthy and secure or not.

In 1994, Naor and Shamir [12] first presented a novel secret sharing scheme called Visual Cryptography (VC) which differs from the traditional cryptography. It divides a black and white image into $\mathrm{n}$ shares. Among those shares, any k or more shares are stacked and then the original image appears, otherwise any less than $\mathrm{k}$ shares can reveal nothing about the original one. The advantage of this Visual Cryptography are very clear in that those complex computations needed in traditional cryptography are redundant and the decryption even does not need the knowledge of cryptography or any help with computer. It only depends on the human's visual system.

The secret image can be decoded by directly superimposing two share images without any extra computation. Each of the shares consists of random pixels and of course meaningless. Since Naor and Shamir published their work, many related methods have been developed.Many Visual Cryptographic methods have been developed. One of the methods of 
VC is Extended Visual Cryptography (EVC). EVC is the VC Scheme where the shares produced are meaningful. This scheme prevents the shares being meaningless and there by prevents revealing the existence of secrets to attackers.

Until now various techniques have been used in EVC. However, it is seen that machine learning techniques have not been used in EVC. Neural networks have found its applications in various fields of science and are effective machine learning tool. Artificial Neural Network (ANN) is defined as: A computing system made up of a number of simple, highly interconnected processing elements, which process information by their dynamic state response to external inputs.

ANNs are processing devices (algorithms or actual hardware) that are loosely modelled after the neuronal structure of the mammalian cerebral cortex but on much smaller scales. A large ANN might have hundreds or thousands of processor units, whereas a mammalian brain has billions of neurons with a corresponding increase in magnitude of their overall interaction and emergent behaviour. Although the mathematics involved with neural networking is not a trivial matter, a user can rather easily gain at least an operational understanding of their structure and function.

Neural networks are typically organized in layers. Layers are made up of a number of interconnected 'nodes' which contain an 'activation function'. Patterns are presented to the network via the 'input layer', which communicates to one or more 'hidden layers' where the actual processing is done via a system of weighted 'connections'. The hidden layers then link to an 'output layer' where the answer is output. Most ANNs contain some form of 'learning rule' which modifies the weights of the connections according to the input patterns that it is presented with. In a sense, ANNs learn by example as do their biological counterparts. There are many different kinds of learning rules used by neural networks

Depending on the nature of the application and the strength of the internal data patterns you can generally expect a network to train quite well. This applies to problems where the relationships may be quite dynamic or non-linear. ANNs provide an analytical alternative to conventional techniques which are often limited by strict assumptions of normality, linearity, variable independence etc. Because an ANN can capture many kinds of relationships it allows the user to quickly and relatively easily model phenomena which otherwise may have been very difficult or impossible to explain otherwise.

The advantage of Neural Network is that it is a powerful data-modeling tool, which has the ability to recognize patterns even if there is no functional relationship between input and output. We have tried to explore the possibility of the use of Neural Network for EVC.

The most commonly used Neural Network model is the Back Propagation Network (BPN) which is a multilayer feed forward network. Feed forward or Back Propagation Network has a number of layers. First a layer has all its inputs connected to either a preceding layer or the inputs from the external world, but not both within the same layer. A layer has all its outputs connected to either a succeeding layer or the outputs to the external world, but not both within the same layer. Next, multiple layers are then arrayed one succeeding the other so that there is an input layer, multiple intermediate layers and finally an output layer, as in Figure 1.

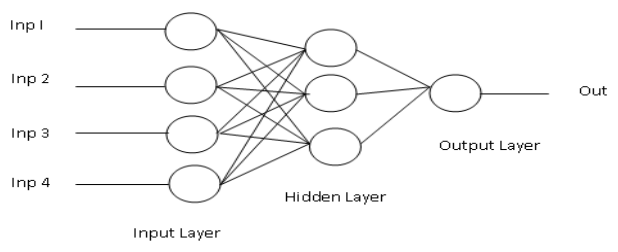

Figure 1. Structure of Feed Forward Network 
Intermediate layers, that are those that have no inputs or outputs to the external world, are called hidden layers. Back propagation neural networks are usually fully connected. This means that each neuron is connected to every output from the preceding layer or one input from the external world if the neuron is in the first layer and, correspondingly, each neuron has its output connected to every neuron in the succeeding layer. Generally, the input layer is considered a distributor of the signals from the external world. Hidden layers are considered to be categorizers or feature detectors of such signals. The output layer is considered a collector of the features detected and producer of the response. While this view of the neural network may be helpful in conceptualizing the functions of the layers, you should not take this model too literally as the functions described may not be so specific or localized. This paper proposes an EVCS using Feed Forward Network.

This paper is organized as follows: Section II deals with the related works. The proposed method is described in Section III. Section IV gives the experimental results, followed by the discussion in Section V. This paper is concluded in Section VI

\section{Related Works}

A brief survey of the related work in the area of visual cryptography is presented in this section. In 1994, Naor and Shamir [12] suggested a cryptography method namely Visual Cryptography. The simplest access structure is the 2 out of 2 scheme where the secret image is encrypted into 2 shares of random binary pattern as shown in Figure 2.

\begin{tabular}{|c|c|c|}
\hline Secret Pixel & $\square$ White Pixel & Black Pixel \\
\hline Share S1 & $\mathbf{B}$ \\
\hline $\begin{array}{c}\text { Share S2 } \\
\text { Stacked } \\
\text { Image }\end{array}$ & $\mathbf{G}$ \\
\hline
\end{tabular}

Figure 2. $(2,2)$ VCS Code Book

According to the color black or white of a secret pixel, the white or black group in the code book is determined. Then one set $2 \times 2$ sub- pixels should be chosen from six code words randomly to generate shares $\mathrm{S} 1$ and $\mathrm{S} 2$. The processed image will be two $2 \mathrm{Nx} 2 \mathrm{~N}$ shares. Stacking the two shares together, we can reveal the secret image. However the secret image can only be black and white images.

Verheul et al. [18] proposed a (k, n)-threshold color visual secret sharing scheme based on pixel expansion for $\mathrm{p}$ color images. Each pixel is expanded to $\mathrm{p}$ sections, and each section is divided into $\mathrm{p}$ sub pixels and can produce $\mathrm{n}$ shares with $\mathrm{p}$ sections. When the $\mathrm{k}$ shares are stacked together, the p-color secret image is revealed. If $\mathrm{p}$ is large, then the pixel expansion is great too. Unfortunately, this scheme tends to produce many blocks with large numbers of black sub pixels when revealing the secret image; in other words, the visual quality is a weakness. Besides, the shares are meaningless. In order to reduce the expansion size of the secret pixel, Yang and Laih [19] proposed a c-color (k, n) - threshold visual secret sharing scheme. Their scheme can indeed reduce the number of black sub pixels effectively. The pixel expansion of this scheme is $\mathrm{c} \times \mathrm{m}$, where $\mathrm{c}$ is the number of colors in the secret image, and $m$ is the pixel expansion size of each color. At less pixel expansion, this scheme improves the visual quality of the revealed secret image. 
Shyu [17] proposed an efficient c-color $(\mathrm{k}, \mathrm{n})$-threshold visual secret sharing scheme and has further improved the pixel expansion to maintain good visual quality of the revealed secret image. Hou [7] proposed three color Visual Cryptography methods where the color secret image is decomposed into three separate images that are respectively colored Cyan (C), Magenta (M) and Yellow (Y). Then the halftone technique is used to translate the three color images into halftone images. Finally, by combining the three halftone images, a color halftone image can be generated. The color halftone image takes eight different colors to display: Cyan, Magenta, Yellow, Black, Red, Green, Blue and White. The three methods proposed take the color halftone image as the secret image. Each block of the two shares will be composed of $\mathrm{C}, \mathrm{M}, \mathrm{Y}$ and $\mathrm{W}$. The secret image can be readily recognized visually when the two shares are stacked together.

However, in spite of all the advancements made in the above schemes, the shares produced by the above methods are meaningless. The Visual Cryptography methods all have the disadvantage that the shares consisting of random pixel patterns do not take any visual information and may lead to suspicion of secret information encryption. Shares showing meaningful images are more desirable in terms of the steganography aspect. To alleviate this problem, Ateniese et al. [2] developed the method of Extended Visual Cryptography (EVC). In EVC, the shares not only contain the secret information, but are also themselves meaningful halftone images.

Hsien $\mathrm{Chu} \mathrm{Wu}$ et al. [8] proposed a method that uses four main procedures. The first procedure is color halftone transformation, where the color image is transformed to a color halftone image. The second procedure, pixel extraction process, extracts pixels from the color halftone image. Third is the encoding process. To generate the shares, two $\mathrm{N} \times \mathrm{N}$ cover images are used to encode the $\mathrm{N} \times \mathrm{N}$ secret image and make two $2 \mathrm{~N} \times 2 \mathrm{~N}$ shares. Finally, during the decoding procedure, the secret image can be easily reconstructed by stacking the two shares. There are two coding tables referred to in the encoding procedure: Cover Coding Table (CCT) and Secret Coding Table (SCT). As the names suggest, CCT is responsible for the encoding of the cover image, and SCT, on the other hand, is used to encode the secret image.

The size of the overlapped image is doubled because of pixel expansion which is overcome in the proposed method. In the proposed method, the size of the two shares as well as the overlapped image is same as that of the original cover and secret images. Tai-Wen Yue \& Suchen Chiang proposed a neural network approach for visual cryptography. The Neural Network is called as Quantum Neural Network, which is a generalized version of Hopfield NN model.

Ge Song, Changgen Peng and Xuelan Miao [5] proposed a method that uses a pi-sigma neural network which is a class of two layer feed forward network. Region based segmentation is used to produce the shares. The target image in the above methods is a gray scale image and it produces shares that are meaningless. All of these existing systems have the drawback of pixel expansion. Further the size of the image gets doubled because of which memory space requirement is also more.

Shyong Jian Shyu and Hung-Wei Jiang [16] proposed a region incrementing visual cryptographic scheme in which linear programming is used. Though the pixel expansion is minimised, the computation time is more for large images.

Shyong Jian Shyu [15] proposed a random grid structure for a general access structure. Kai-Hui Lee and Pei-Ling Chiu [9] proposed an approach that consists of two phases. In the first phase, based on a given access structure, we construct meaningless shares using an optimization technique and the construction for conventional VC Schemes. In the second phase, cover images are added in each share directly by a stamping algorithm. Pixel expansion is reduced to some extent. 
In order to overcome all these limitations, in this paper, an Extended Visual Cryptography Scheme using Feed Forward Network that produces the shares whose size is same as that of the original images is proposed.

\section{Proposed Method}

This section provides a brief description of steps to be followed in EVCS using Feed forward Network.

The input for this proposed methods are two color images which are used as cover images and one image that is the secret image. This secret image is divided into two shares. The two shares are embedded in the two cover images. The three images should be of the same size. The output produced will be two shares that have the secret image embedded in it. This ouptut produced also will be of the same size. When the two shares are overlapped the secret image can be obtained. The main steps involved in the proposed method are diagrammatically shown in the Figure 3. It is explained in the following section.

\subsection{Color Halftone Transformation}

There are three steps in the color halftone transformation phase.

3.1.1. Image Resizing: In this step the three images are halved from its original size. For example if the size of the image is $256 \times 256$, then it is reduced to $128 \times 128$. The storage space as well as the encoding time gets reduced as the image is reduced to half their size.

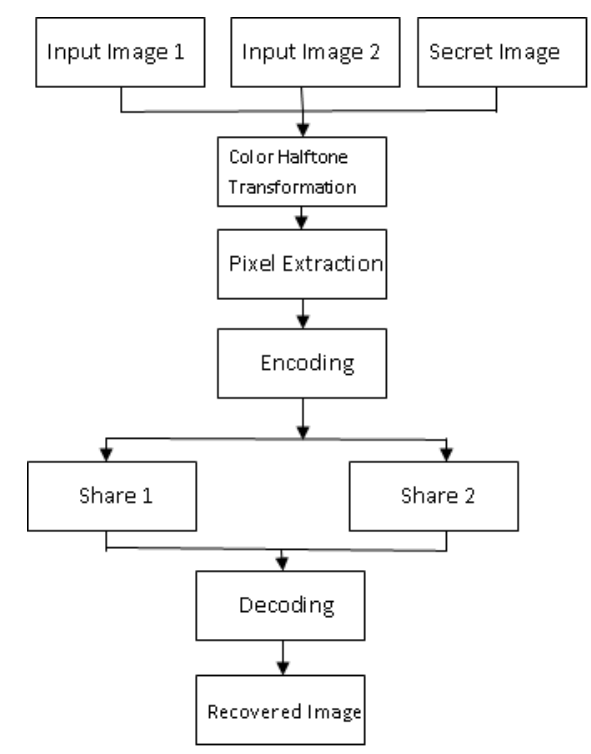

Figure 3. Block Diagram of Extended Visual Cryptography

3.1.2. Color Decomposition: The three images are decomposed into three separate images as Cyan, Magenta and Yellow. This is done as a pre-processing step for halftoning.

3.1.3. Halftoning: Halftoning is applied for the images obtained from the color decomposition step. Halftoning is the method that produces an image by varying the pattern of dots to produce different shades and colors. Error diffusion Halftoning is applied as it produces much higher quality. Halftone is applied on each monochrome image obtained from the previous step. Each monochrome image is treated as gray-scale image and transformed 
into binary image. Then the three binary images are combined to produce a single halftone image. This process is applied for all three images namely Secret image, Cover Image 1, and Cover Image 2

\subsection{Pixel Extraction}

The output of halftone step is a halftoned image. Then pixel extraction procedure is done to extract important pixel from the images. For this either the odd numbered rows or columns can be selected. In this method, odd numbered columns are selected. If the pixel size is 256, then the size of the extracted image is $128 \times 256$.

\subsection{Encoding}

In the encoding procedure the Secret Image is divided into two shares and the two shares are embedded in the two Cover Images namely Cover Image1 and Cover Image2. For the encoding procedure, two tables namely Cover Coding Table (CCT) and Secret Coding Table (SCT) are used. There are three steps in encoding namely Secret Image Coding, Cover Image Coding and Share Production

3.3.1. Secret Image Coding: The Secret Image is given as input to this step. This Secret Image is coded with the Secret Coding Table (SCT). The SCT is used in the same way as it was used in Hou's [2] second method as given in the Table II. The Back Propagation Network is first trained with this Secret Coding Table. The inputs to the Back Propagation Network are the eight pixel values. The neural network is trained to produce the blocks of Share 2 with four pixels namely Cyan, Magenta, Yellow and White. Once the network starts producing the expected output, then it can be used to process the secret image.

For each pixel of the secret image, the following process is done. First, for Share1, $2 \times 2$ block is built by permuting the four pixels $\mathrm{C}, \mathrm{M}, \mathrm{Y}$ and $\mathrm{W}$. Then, the corresponding block for share 2 is selected from the Table 1.

Table 1. Secret Coding Table

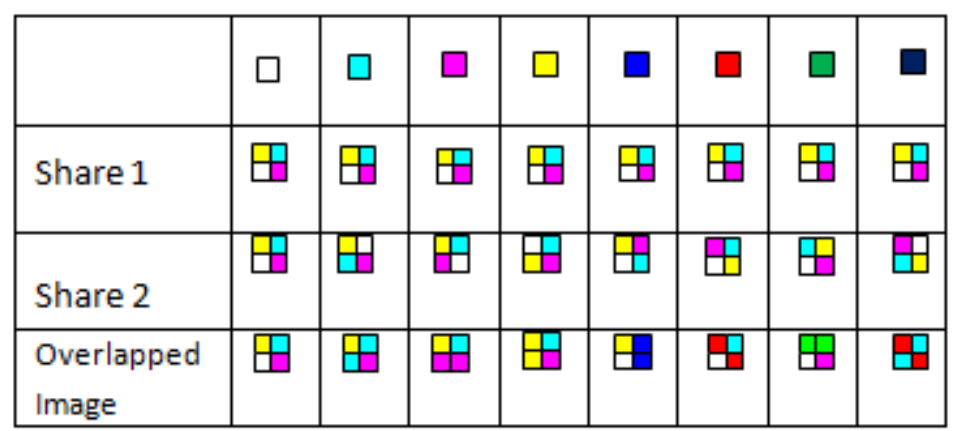

For example if the pixel in the secret image is blue, Share1 gets a block with four pixels as Yellow, Cyan, White and Magenta. According to the Secret Coding table, the block in Share2 gets four pixels as Yellow, Magenta, White and Cyan. When all the pixels are processed, the two shares for the secret image are produced.

3.3.2. Cover Image Coding: The two cover images namely Cover Image 1 and Cover Image 2 are given as input to the Cover Image Coding procedure. Cover Coding Table as given in Table 3 is used to code the cover image. This step generates $2 \times 2$ blocks from the cover 
images. The neurons of the Feed Forward Network are trained to obtain the two blocks of pixel as given in Table 2 .

Table 2. Cover Coding Table

\begin{tabular}{|c|c|c|c|c|c|c|c|c|}
\hline & $\square$ & 口 & 口 & 口 & घ & 口 & 口 & घ \\
\hline 口 & 田田 & 口曰 & 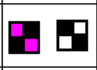 & 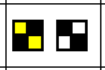 & E & 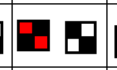 & 田田 & \\
\hline$\square$ & 田田 & 田田 & 田田 & $\mathbf{⿴ 囗 十 \boldsymbol { O }}$ & 回 & 100 & D & $\square$ \\
\hline$\square$ & OD & 田田 & D日 & 由 & DE & 田 & DQ & 10 \\
\hline$\square$ & 田 & 田田 & 田田 & 田田 & 回 & 田 & 田田 & 10 \\
\hline 口 & 田 & & 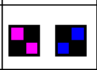 & $\mathbf{⿴ 囗 十 力}$ & & 10 & G日 & \\
\hline$\square$ & 田 & U & 田 & 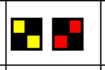 & 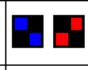 & & a日 & 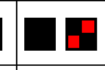 \\
\hline 口 & 田田 & 田 & 8日 & $\mathbf{B O}$ & Et & 由日 & 田田 & $\theta$ \\
\hline & & & & & & & 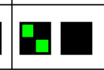 & \\
\hline
\end{tabular}

The inputs to the Feed Forward Network are the combination of the pixel values in the two cover images. The outputs of the Feed Forward Network are two blocks with four pixels each. Thus the Feed Forward Network is trained to produce the blocks as given in the Cover Coding Table. Once the neural network has been trained, then it can be used to produce the two blocks based on the cover images.

3.3.3. Share Production: The two shares produced in the secret image coding step and the blocks produced in the cover image coding step becomes the input for this step. The outputs are two patterns $\mathrm{P} 1$ and $\mathrm{P} 2$ with two regions $\mathrm{R} 1$ and R2.For example, when the pixel in Cover Image 1 is blue and the pixel in Cover Image 2 is green; two blocks (B1 and B2) are produced using Cover Coding Table. These two blocks are then combined with the two blocks (B3 and B4) produced from the secret image to form the two regions (R1 and R2) in the two patterns (P1 and P2).

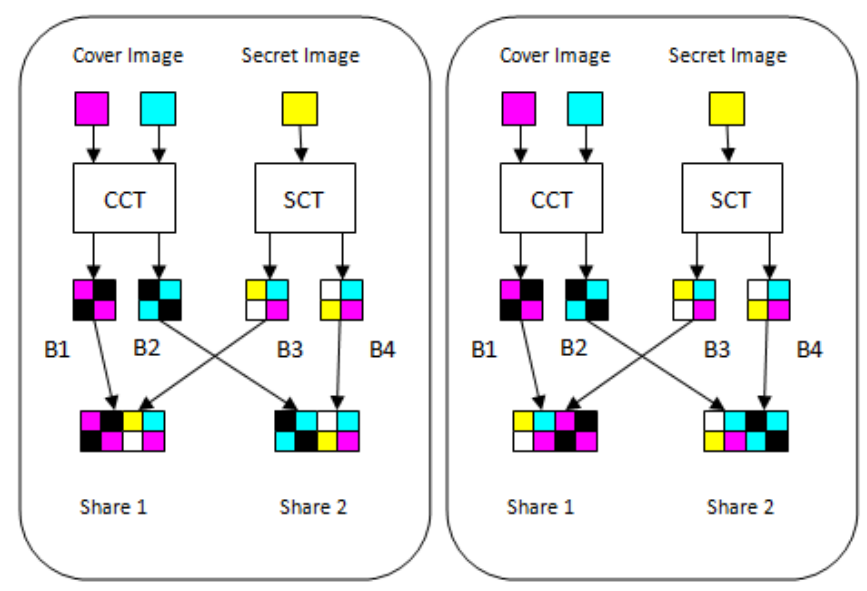

Figure 4. Production of Shares for Odd Pixel and Even Pixel 
This is done based on the position of the pixel in the shares. If the pixel belongs to an odd row, R1 of P1 has B1 and R2 of P1 has B3. Similarly R1 of P2 has B2 and R2 of P2 has B4. If the pixel belongs to even row, R1 of P1 has B3 and R2 of P1 has B1. Similarly R1 of P2 has $\mathrm{B} 4$ and $\mathrm{R} 2$ of $\mathrm{P} 2$ has $\mathrm{B} 2$. When all the blocks have been processed, the production of shares is completed. The complete share production process is shown in Figure 4.

\subsection{Decoding}

Decoding is the procedure in which the two shares are overlapped to produce the secret image. Here no computation is required to decode the original image. Some of the blocks become black after overlapping. However that does not affect the secret image. It improves the contrast of the secret image and makes the image clearer.

\section{Experimental Results}

Two cover images and one secret image of the same size are taken. As the images are resized in the first step, the shares produced in the encoding step are of the same size. When the two shares are overlapped we get the secret image which is of the same size and of better quality as we have used Feed Forward Network. No mathematical calculations are needed for the decoding step.

When the first block of Share 1 is overlapped with the first block of Share 2, it would produce the pixel of the secret image whereas the second block would produce a block consisting of black pixel. In this way, the whole secret image is obtained when the two shares are overlapped. Figure 5a to Figure 5d shows the encoding and decoding of Lena image of various sizes. Two shares are produced in the encoding step. Figure $6 a$ to Figure $7 d$ shows the encoding and decoding of Peppers and Boat images of various sizes respectively.

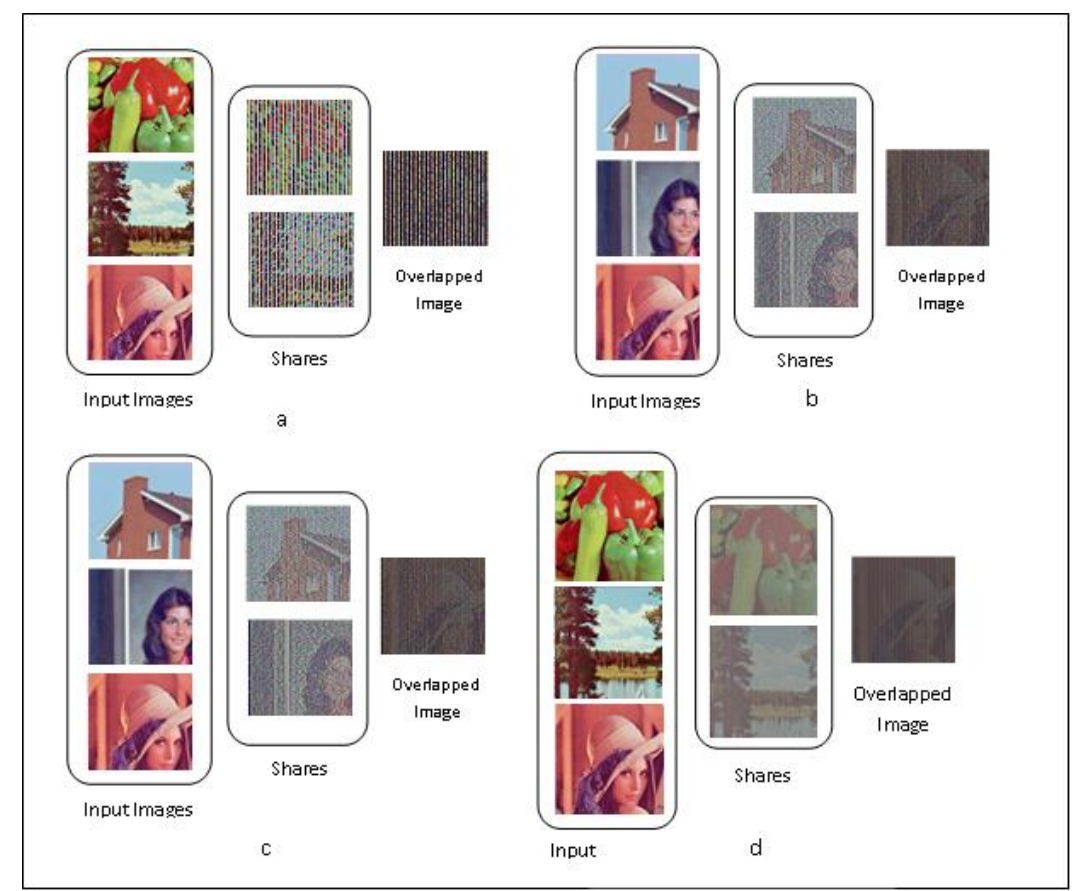

Figure 5. a-d Encoding and Decoding of Lena Image of Sizes 128x128, 256x256, $512 \times 512,1024 \times 1024$ 


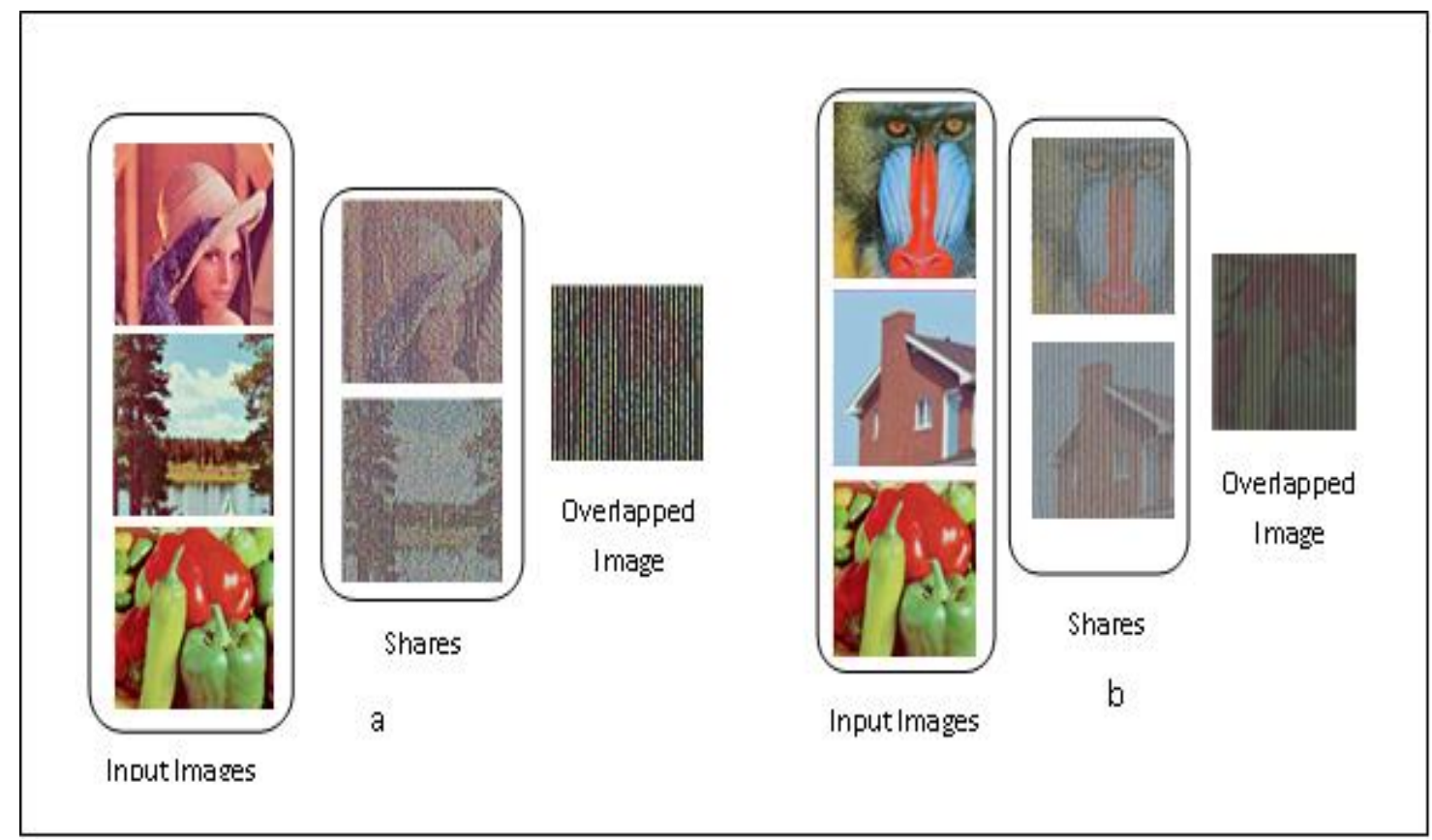

Figure 6. a,b Encoding and Decoding of Pepper Image of Sizes 128x128, $256 \times 256$

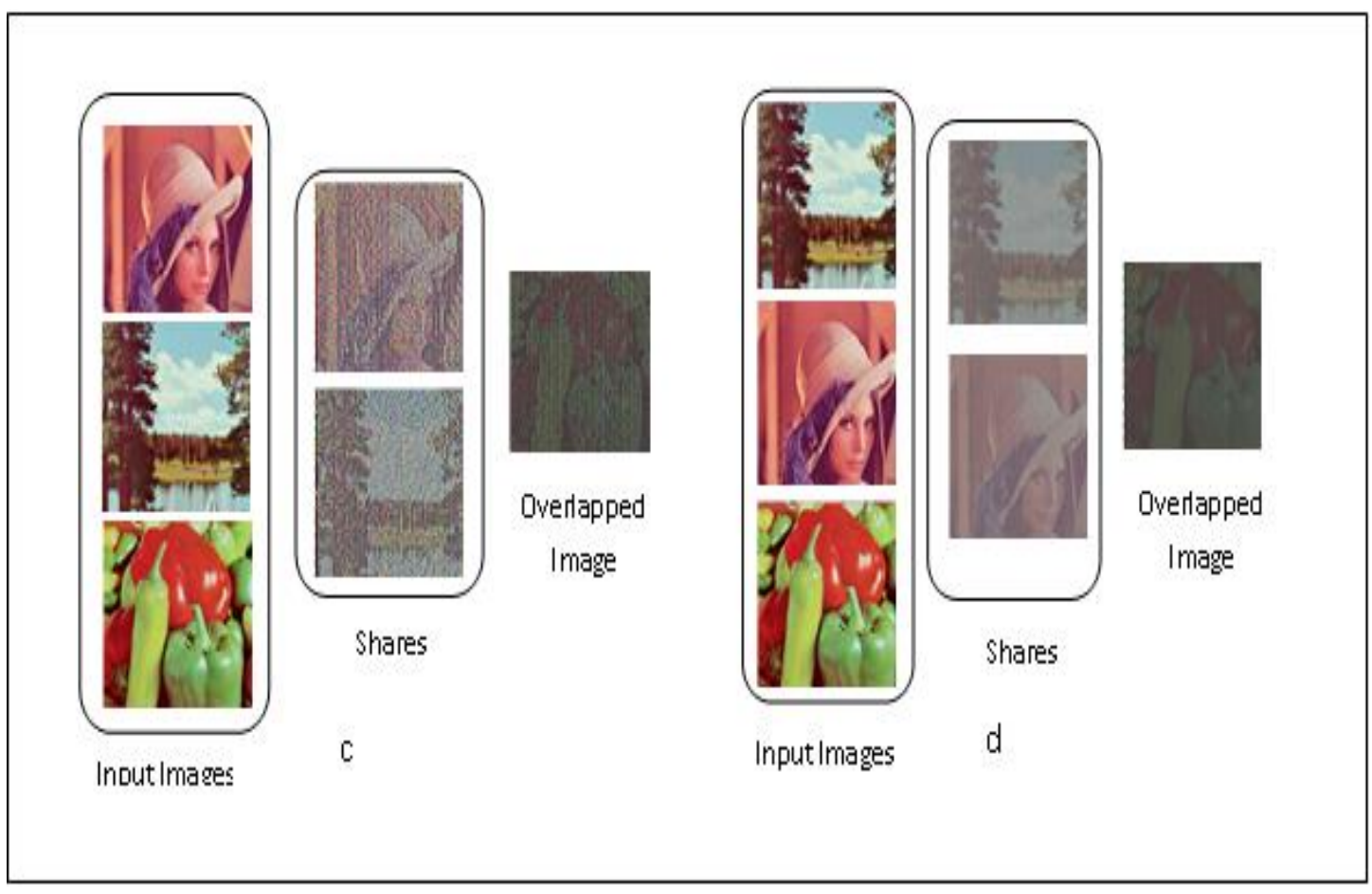

Figure 6. c, d Encoding and Decoding of Pepper Image of 512x512, 1024×1024 


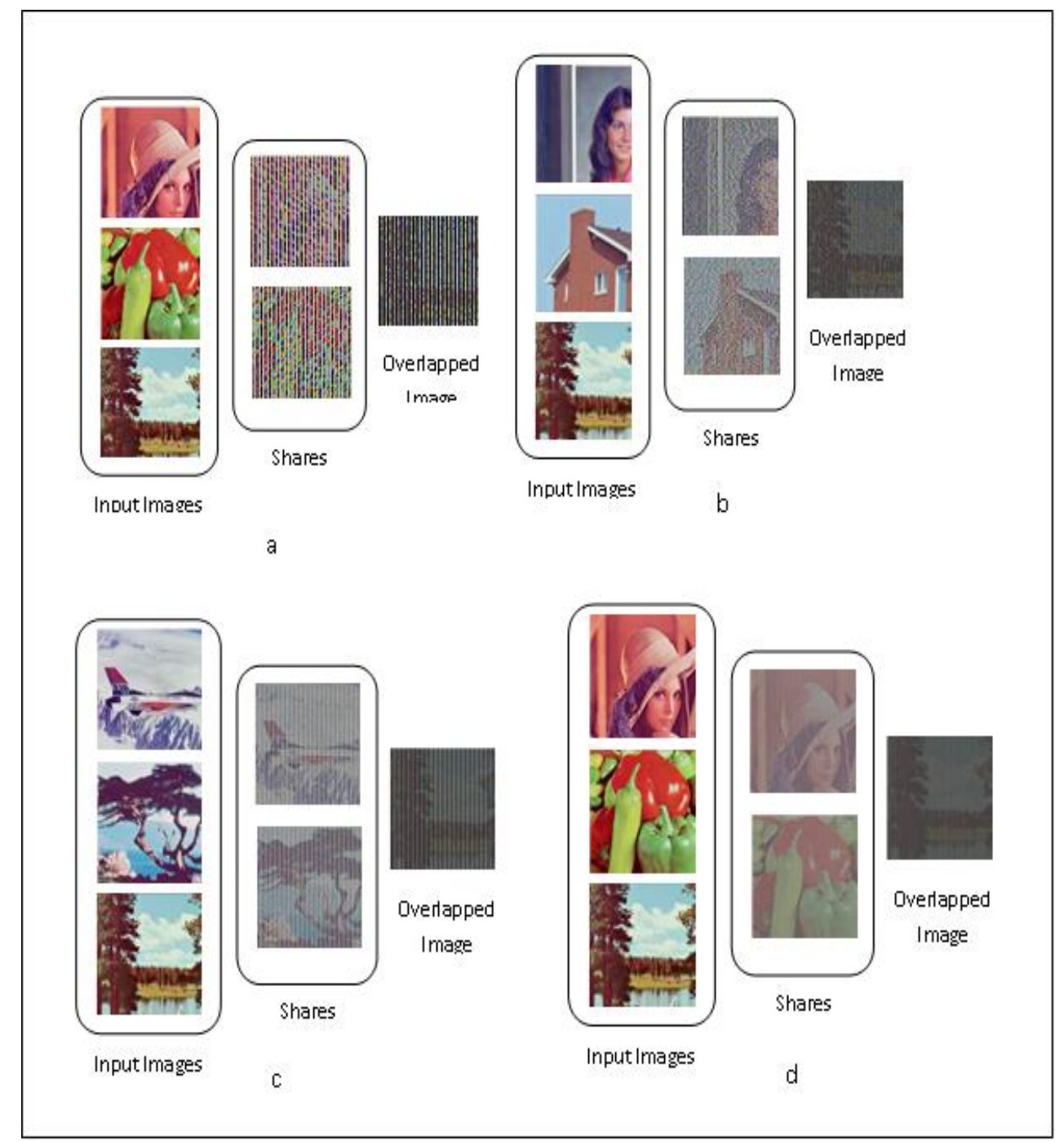

Figure 6. a-d Encoding and Decoding of Pepper Image of Sizes 128x128, $256 \times 256,512 \times 512$, and $1024 \times 1024$

\section{Discussion}

The probability of the secret image block being guessed correctly can be calculated by the formula $(1 / \mathrm{p}) \mathrm{N} / 2 \mathrm{xN}$, where $\mathrm{p}$ is the number of block combination and $\mathrm{N} / 2 \mathrm{xN}$ is the size of the extracted secret image. If the pixel is blue, the block combinations that produce blue are8. Hence the probability of the extracted secret image being guessed correctly is extremely low. This way, with each block randomly produced, our scheme makes it extremely difficult for an attacker to figure out what the secret image is.

PSNR and Correlation coefficient are taken to validate the proposed method. For the three images namely Peppers, Lena and Boat, PSNR value of these images with sizes $128 \times 128$, 256x256, 512x512and 1024x1024 is calculated. The PSNR values of these images are given in the Table 3. 
Table 3. PSNR Values of Overlapped Images

\begin{tabular}{|c|c|c|}
\hline Image & Size & $\begin{array}{c}\text { PSNR } \\
\mathbf{( d b )}\end{array}$ \\
\hline \multirow{4}{*}{ Lena } & $128 \times 128$ & 41.1897 \\
\cline { 2 - 3 } & $256 \times 256$ & 35.1675 \\
\cline { 2 - 3 } & $512 \times 512$ & 29.1488 \\
\cline { 2 - 3 } & $1024 \times 1024$ & 23.127 \\
\hline \multirow{4}{*}{ Pepper } & $128 \times 128$ & 41.2826 \\
\cline { 2 - 3 } & $256 \times 256$ & 35.2483 \\
\cline { 2 - 3 } & $512 \times 512$ & 29.2290 \\
\cline { 2 - 3 } & $1024 \times 1024$ & 23.2085 \\
\hline \multirow{4}{*}{ Boat } & $128 \times 128$ & 41.2016 \\
\cline { 2 - 3 } & $256 \times 256$ & 35.1700 \\
\cline { 2 - 3 } & $512 \times 512$ & 29.1443 \\
\cline { 2 - 3 } & $1024 \times 1024$ & 23.1260 \\
\hline
\end{tabular}

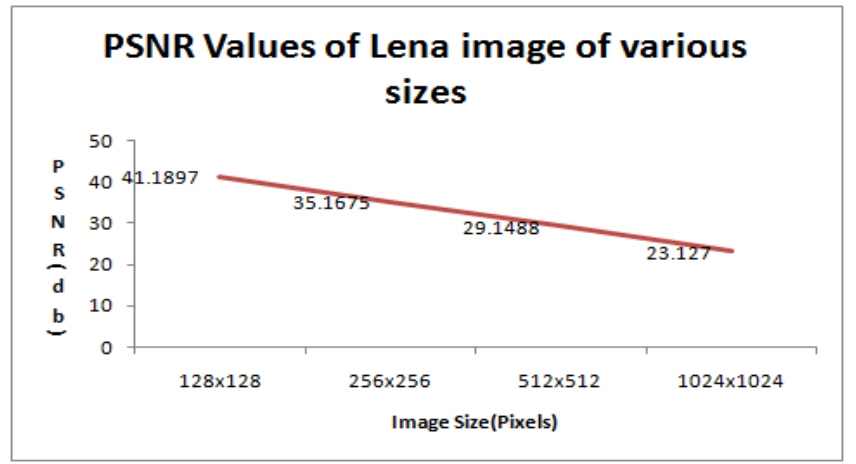

\section{Figure 8. Comparison of PSNR Values of Lena Images}

From the Table 3, it can be observed that PSNR value decreases as the size of the image increases. This shows that the overlapped image is of better quality when compared to the existing systems.

A comparison is made between the PSNR values of the three images namely Lena, Pepper, and Boat of sizes namely $128 \times 128,256 \times 256,512 \times 512$ and $1024 \times 1024$. This is shown in Figure 8, Figure 9 and Figure 10

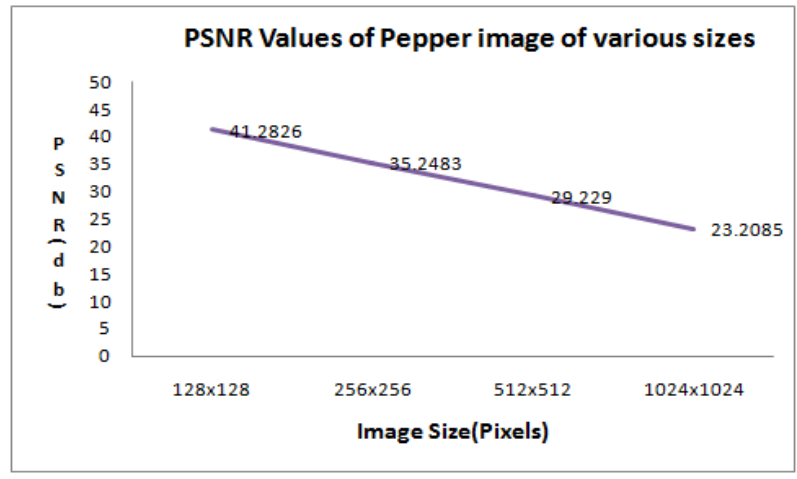

Figure 9. Comparison of PSNR Values of Pepper Images 


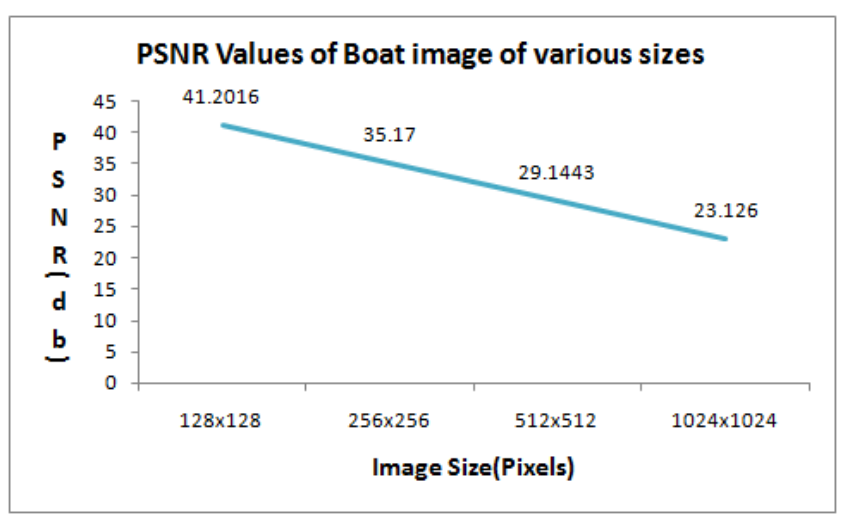

Figure 10. Comparison of PSNR Values of Boat Images

Correlation is a method of identifying the degree of relationship between two sets of values. Karl Pearsons correlation coefficient reveals the dependency or independency between the variables.

The value of correlation coefficient ranges from -1 to +1 . If the value of correlation coefficient is -1 , the two sets are inversely related. If the value is 1 , then the variables are completely related. So if the correlation coefficient of the original image and the output image is nearer to +1 , then the overlapped image is of higher clarity as shown in the Table 4 .

Table 4. Correlation Coefficient of Overlapped Images

\begin{tabular}{|c|c|c|}
\hline Image & Size & $\begin{array}{c}\text { Correlation } \\
\text { coefficient }\end{array}$ \\
\hline \multirow{4}{*}{ Boat } & $128 \times 128$ & 0.5887 \\
\cline { 2 - 3 } & $256 \times 256$ & 0.5594 \\
\cline { 2 - 3 } & $512 \times 512$ & 0.5621 \\
\cline { 2 - 3 } & $1024 \times 1024$ & 0.5599 \\
\hline \multirow{4}{*}{ Pepper } & $128 \times 128$ & 0.5499 \\
\cline { 2 - 3 } & $256 \times 256$ & 0.5511 \\
\cline { 2 - 3 } & $512 \times 512$ & 0.5515 \\
\cline { 2 - 3 } & $1024 \times 1024$ & 0.5422 \\
\hline \multirow{4}{*}{ Lena } & $128 \times 128$ & 0.5600 \\
\cline { 2 - 3 } & $256 \times 256$ & 0.5602 \\
\cline { 2 - 3 } & $512 \times 512$ & 0.5600 \\
\cline { 2 - 3 } & $1024 \times 1024$ & 0.5601 \\
\hline
\end{tabular}

The next comparison with the existing system is based on the memory space required for the overlapped image. This is shown in Table 5. From this table it can observed that the 
memory space required for storing the overlapped image in the proposed system is less when compared to the existing system. The graph below in Figure 11 shows the comparison of the existing system with proposed system based on the memory space required to store the overlapped image.

Table 5. Comparison of the Proposed System with Existing System based on Memory Space

\begin{tabular}{|c|c|c|c|c|}
\hline \multirow{3}{*}{ Image } & \multirow{3}{*}{ Size } & \multicolumn{3}{|c|}{ Memory Space Required for } \\
\hline & & \multirow{2}{*}{$\begin{array}{l}\text { Secret } \\
\text { Image }\end{array}$} & \multicolumn{2}{|c|}{ Overlapped Image In } \\
\hline & & & $\begin{array}{l}\text { Existing } \\
\text { Systems }\end{array}$ & $\begin{array}{c}\text { Proposed } \\
\text { System }\end{array}$ \\
\hline \multirow{3}{*}{ Lena } & $128 \times 128$ & $48.6 \mathrm{~KB}$ & $114 \mathrm{~KB}$ & $28.7 \mathrm{~KB}$ \\
\hline & $256 \times 256$ & $193 \mathrm{~KB}$ & $461 \mathrm{~KB}$ & $114 \mathrm{~KB}$ \\
\hline & $512 \times 512$ & $768 \mathrm{~KB}$ & $1.79 \mathrm{MB}$ & $457 \mathrm{~KB}$ \\
\hline \multirow{3}{*}{ Pepper } & $128 \times 128$ & $48.6 \mathrm{~KB}$ & $112 \mathrm{~KB}$ & $27.9 \mathrm{~KB}$ \\
\hline & $256 \times 256$ & $193 \mathrm{~KB}$ & $449 \mathrm{~KB}$ & $112 \mathrm{~KB}$ \\
\hline & $512 \times 512$ & $768 \mathrm{~KB}$ & $1.75 \mathrm{MB}$ & $449 \mathrm{~KB}$ \\
\hline \multirow{3}{*}{ Boat } & $128 \times 128$ & $48.6 \mathrm{~KB}$ & $112 \mathrm{~KB}$ & $28.0 \mathrm{~KB}$ \\
\hline & $256 \times 256$ & $193 \mathrm{~KB}$ & $450 \mathrm{~KB}$ & $112 \mathrm{~KB}$ \\
\hline & $512 \times 512$ & $768 \mathrm{~KB}$ & $1.76 \mathrm{MB}$ & $450 \mathrm{~KB}$ \\
\hline
\end{tabular}

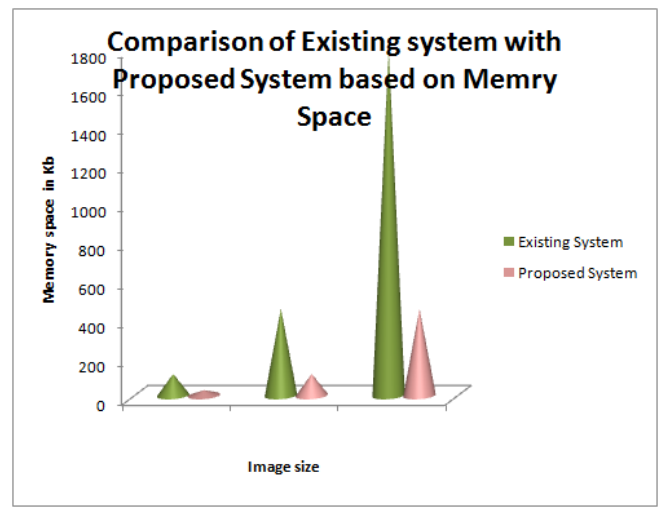

Figure 11. Comparison of Existing System with Proposed System on Memory

\section{Conclusion}

Thus an efficient way to improve the method of producing shares in Extended Visual Cryptography is proposed in this paper. As the image is resized, the secret image obtained is of the same size as that of the original image. As Feed Forward Network is used to generate the shares, the image is of higher quality as clearly depicted by the experimental results.

Further, this method can be extended to reduce the pixel expansion, so that the image quality can still be improved. 


\section{References}

[1] M. A. Reddy, P. S. Bala and G. Aghila, "Comparison of Visual Cryptographic schemes", International Journal of Engineering Science and Technology, vol. 3, (2011).

[2] Ateniese, C. Blundo, A. de Santis, and D. Stinson, "Visual Cryptography for General Access Structures," Information and Computation, vol. 129, no. 2, (2010).

[3] F. Liu, C. Wu and X. Lin," Step Construction of Visual Cryptography Schemes", IEEE Transactions on Information Forensics and Security, vol. 5, (2010).

[4] F. Liu and C. Wu, "Embedded Extended Visual Cryptography Schemes", IEEE Transactions on Information Forensics and Security, vol. 6, no. 2 (2011).

[5] G. Song, C. Peng and X. Miao, "Visual Cryptography Scheme Using Pi-sigma Neural Networks", Proceedings of the 2008 International Symposium on Information Science and Engineering, (2008), pp. 679682.

[6] G. Krishnan and S. Loganathan, D, "Color Image Cryptography Scheme Based on Visual Cryptography", Proceedings of 2011 International Conference on Signal Processing, Communication, Computing and Networking Technologies, (2011), pp. 404-407.

[7] Y. C. Hou, "Visual cryptography for color images", Pattern Recognition, vol. 36, (2003).

[8] H.-C. Wu, H.-C. Wang and R.-W. Yu, "Color Visual Cryptography Scheme Using Meaningful Shares", Proceedings of the Eighth International Conference on Intelligent Systems Design and Applications, (2008), pp. 173-178.

[9] K.-H. Lee and P.-L. Chiu, "An Extended Visual Cryptography Algorithm for General Access Structures", IEEE Transactions on Information Forensics and Security, vol. 7, no. 1, (2012).

[10] I. Kang, G. R. Arce and H.-K. Lee, "Color extended visual cryptography using error diffusion", Proceedings of ICASSP 2009, (2009), pp. 1473-1476.

[11] K. Kumari and S. Bhatia, "Multi-pixel Visual Cryptography for color images with Meaningful Shares", International Journal of Engineering Science and Technology, vol. 2, (2010).

[12] M. Naor and A. Shamir, "Visual cryptography," Advances in Cryptology - EUROCRYPT'94, (1995), pp. 112.

[13] T. Rajitha, P. P. Kumar and V. Laxmi "Construction of Extended Visual Cryptography Scheme for Secret Sharing", International Journal of Computer Science and Network (IJCSN), vol. 1, no. 4, (2012).

[14] P. S. Revenkar, A. Anjum and W. Z. Gandhare, "Survey of Visual Cryptography Schemes", International Journal of Security and Its Applications, vol. 4, (2010).

[15] S. J. Shyu, "Visual Cryptograms of Random Grids for General Access Structures", IEEE Transactions on Circuits And Systems For Video Technology, vol. 23, no. 3, (2013).

[16] S. J. Shyu and H.-W. Jiang, "Efficient Construction for Region Incrementing Visual Cryptography" IEEE Transactions On Circuits And Systems For Video Technology, vol. 22, no. 5, (2012).

[17] S. J. Shyu, "Efficient visual secret sharing scheme for color images", Pattern Recognition, vol. 39, (2006).

[18] E. R. Verheul and H. C. A. van Tilborg, "Constructions and properties of $\mathrm{k}$ out of $\mathrm{n}$ visual secret sharing schemes," Designs, Codes and Cryptography, vol. 11, no. 2, (1997).

[19] C. N. Yang and C. S. Laih, "New colored visual secret sharing schemes," Designs, Codes and Cryptography, vol. 20 , no. $3, \mathbf{( 2 0 0 0 )}$. 